\title{
Microwave-assisted Synthesis Silver Nanoparticles and Their Surface Enhancement Raman Scattering
}

\author{
Liu Faxian ${ }^{1,2}, \quad$ Liu Jie ${ }^{1}, \quad$ Cao Xueling ${ }^{3}$ \\ ${ }^{1}$ Key Laboratory of Carbon Fiber and Functional Polymers, Ministry of Education, Beijing University of Chemical Technology, Beijing 100029, \\ China; ${ }^{2}$ Jilin Petrochemical Company, Jilin 132002, China; ${ }^{3}$ Jilin Institute of Chemical Technology, Jilin 132022, China
}

\begin{abstract}
A one-step microwave-assisted method was used for the synthesis of silver nanoparticles, and the corresponding nature and structure of silver nanoparticles were characterized by UV-Vis absorption spectroscopy, transmission electron microscopy (TEM), and atomic force microscopy (AFM). Raman scattering activities of silver nanoparticles with different reaction time were explored using rhodamine 6G (R6G) as a probe molecule. The results show that the intensity of surface enhancement Raman scattering (SERS) signals could be increased by silver nanoparticles. And then we made a comparison on SERS spectra of R6G studied silver nanoparticles to investigate the effect of surface enhancement Raman. Meanwhile, we studied the mechanism in the process of enhancement by silver nanoparticles.
\end{abstract}

Key words: microwave-assisted method; silver nanoparticles; surface enhancement Raman scattering

Since the surface enhancement Raman scattering (SERS) effect was discovered for the first time by M. Fleischmann ${ }^{[1]}$ in 1974, SERS has attracted considerable attention on both theoretical and experimental investigations ${ }^{[2-9]}$. Recently, single-molecule detection ${ }^{[10-13]}$ should be the most progress, showing enhancement factors up to $10^{14} \sim 10^{15}$ on a single silver nanoparticle. Due to the excellent optical properties, silver nanoparticles have been widely used for SERS.

Different approaches to the synthesis of silver nanoparticles have been reported such as chemical reduction, UV photolysis and gamma irradiation ${ }^{[14]}$. Among them, the chemical reduction was still the most common method. In addition, microwave-assisted heating has been coupled with the chemical reduction as a convenient and energy efficient preparation method of silver nanoparticles. The main advantage of microwave-assisting over conventional heating is rapid and uniform since no high temperatures or high pressures are needed ${ }^{[15,16]}$. Therefore, both nucleation and growth could be controlled easily to produce nano-products with the desired shape or size. Microwave-assisted methods have been widely used for the synthesis of metallic nanoparticles such as gold, silver, palladium and platinum ${ }^{[10,17]}$ Here we reported a one-step microwave-assisted synthesis of silver nanoparticles. Furthermore, we provided a direct application of the silver nanoparticles to the surface enhancement Raman scattering. Meanwhile, silver nanoparticles were produced at three different reaction time, and then we made a comparison on SERS spectra of R6G molecules adsorbed on the three kinds of silver nanoparticles to investigate the effect of surface enhancement Raman.

\section{Experiment}

The silver nitrate was purchased from Sigma and used without further purification. The trisodium citrate was purchased from Beijing Chemical Company. All other reagents were of analytical reagent grade and used as received. The water used was purified through a Millipore system.

The silver nanoparticles were synthesized by BILONCW-1000 ultrasonic microwave synthesizer (Shanghai Bilon Instrument Manufacturing CO.). The $\mathrm{AgNO}_{3}$ aqueous $(10 \mathrm{~mL}$, $10 \mathrm{mmol} / \mathrm{L})$ was added to trisodium citrate aqueous $(10 \mathrm{~mL}$, $10 \mathrm{mmol} / \mathrm{L}$ ) at $37{ }^{\circ} \mathrm{C}$. Two minutes later, the mixture was

Received date: September 14,2016

Foundation item: The National Natural Science Foundation of China (51073011)

Corresponding author: Liu Jie, Ph. D., Professor, College of Materials Science and Engineering, Beijing University of Chemical Technology, Beijing 100029, P. R. China, Tel: 0086-10-64438724, E-mail: liujj_432@163.com 
incubated with the microwave irradiation with indirect response mode for the different time ( $t=8,15,20 \mathrm{~min})$. The solution was centrifuged with $10000 \mathrm{r} / \mathrm{min}$ and then thoroughly rinsed twice in deionized water to obtain silver nanoparticles. The deposit was collected and then subjected to freeze-drying to obtain silver nanoparticles in powder.

The UV-Vis spectra were recorded on a Shimadzu UV-3600 spectrophotometer for solutions with concentration of 10 $\mathrm{mg} / \mathrm{mL}$ silver nanoparticles contained in $1 \mathrm{~cm} \times 1 \mathrm{~cm}$ quartz cuvettes ( $4 \mathrm{~mL}$ volume).

A certain amount of silver nanoparticles was gained with a special copper mesh, then placed and dried under an infrared lamp for $1.5 \mathrm{~h}$. The copper mesh with the sample was placed into the sample tank, the working voltage of JEM-2000EX transmission electron microscopy was adjusted to $200 \mathrm{kV}$, and then transmission electron microscopy images was obtained.

Raman spectra were recorded on Raman spectrometer (Renishaw Invia, UK) equipped with inverse microscope (ZEISS Axiovert 25). The spectral response range of Raman spectra was $500 \sim 1700 \mathrm{~cm}^{-1}$. The spectra were excited by 632.8 $\mathrm{nm}$ line of $35 \mathrm{~mW} \mathrm{He}-\mathrm{Ne}$ laser at $10 \mathrm{~s}$ exposure.

\section{Results and Discussion}

\subsection{UV-Vis spectra of silver nanoparticles}

For the microwave-assisted synthesis of silver nanoparticles, trisodium citrate was reacted with silver nitrate aqueous under extremely basic conditions ( $\mathrm{pH} 8$ ) at $37{ }^{\circ} \mathrm{C}$ by MWI instead of direct heating to maintain the temperature. The growth of silver nanoparticles as a function of reaction condition was followed by UV-Vis spectra. When the initial powers of MWI are varied from 100 to $700 \mathrm{~W}$ and the reaction time is $10 \mathrm{~min}$, the maximum absorption wavelength red-shift gradually with reaction power, ascribed to the slow growth of silver nanoparticles (Fig.1). After $10 \mathrm{~min}$ of MWI at $500 \mathrm{~W}$, the UV-Vis spectra change slightly when the molar ratio of silver nitrate and trisodium citrate varies in the range of $3: 1 \sim 1: 3$ (Fig.2). However, for the molar ratio of 1:3, the maximum absorption wavelength decreases more quickly.

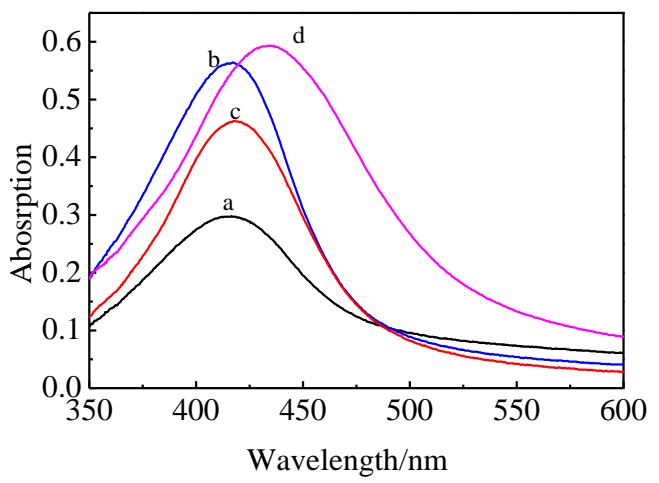

Fig.1 UV-Vis absorption spectra of silver nanoparticles prepared with different MWI powers (a- $100 \mathrm{~W}, \mathrm{~b}-300 \mathrm{~W}, \mathrm{c}-500 \mathrm{~W}$, d- $700 \mathrm{~W}$ )

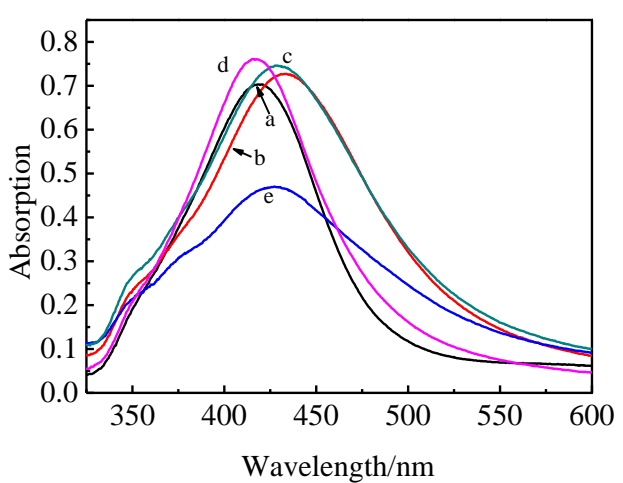

Fig.2 UV-Vis absorption spectra of silver nanoparticles prepared with different molar ratios of $\mathrm{AgNO}_{3}$ and trisodium citrate (a- $3: 1$, b- $2: 1$, c- $1: 1$, d- $1: 2$, and e- $1: 3$ )

Fig. 3 provides the UV-Vis spectra of three original silver nanoparticles obtained by the reduction method, namely for the power setting of $500 \mathrm{~W}$ silver nitrate and trisodium citrate (the molar ratio was 1:1) were mixed and heated for different time $(t=8,15,20 \mathrm{~min})$. These spectra indicate that metallic silver nanoparticles have different sizes after different reaction time. At reaction time of $8,15,20 \mathrm{~min}$, the plasmon resonance peaks appear at 408, 423, $461 \mathrm{~nm}$, respectively (Fig.3). It is shown that the size of silver nanoparticles increases gradually with the increasing of the reaction time. So we could adjust the reaction conditions to prepare different sizes of silver nanoparticles.

\subsection{TEM images of silver nanoparticles}

TEM images of silver nanoparticles are shown in Fig.4. It is obvious that silver nanoparticles are uniformly spherical in shape and well-dispersed. The mean size of these nanoparticles is approximately $60 \mathrm{~nm}$, which is in agreement with that of the UV-Vis spectrum. And with the increase in reaction time, the size of the silver nanoparticles was larger, but when the reaction time is $20 \mathrm{~min}$, some certain aggregation occurs in silver nanoparticles.

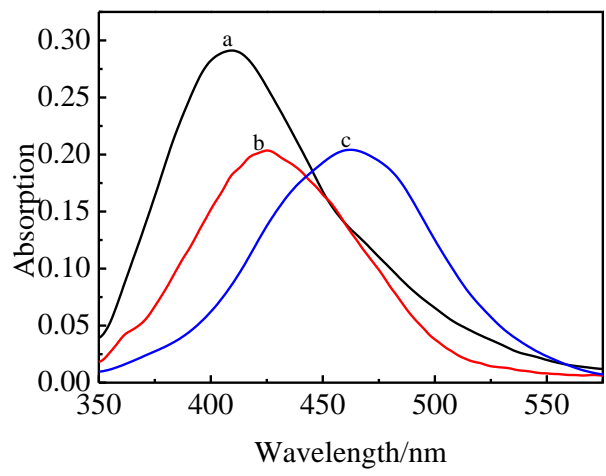

Fig.3 UV-Vis absorption spectra of silver nanoparticles prepared with different heating time (a- $8 \mathrm{~min}, \mathrm{~b}-15 \mathrm{~min}$, and c- 20 $\min )$ 


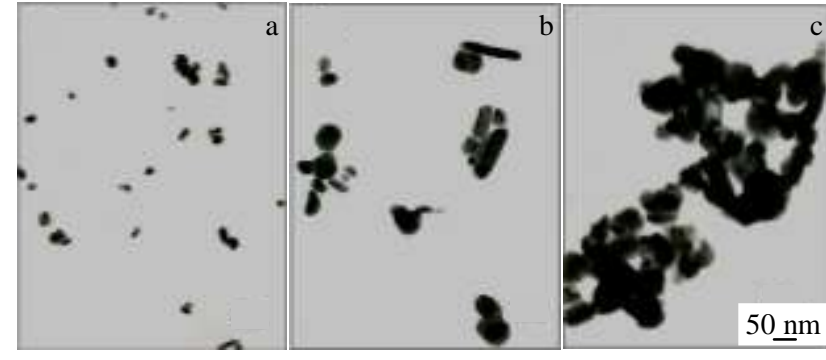

Fig.4 TEM images of silver nanoparticles prepared under microwave irradiation with different heating time: (a) $8 \mathrm{~min}$, (b) 15 min, and (c) $20 \mathrm{~min}$

\subsection{AFM analysis of silver nanoparticles}

The sample was subsequently examined by AFM. As shown in Fig.5, silver nanoparticles have a diameter of around $60 \mathrm{~nm}$. This is consistent with the results obtained by absorption spectra and TEM.

These experimental results suggest that the size of silver nanoparticles depends on the reaction time. Therefore, it is possible to control the size of silver nanoparticles by varying the microwave heating time as previously reported in many literature ${ }^{[12,17]}$.

\subsection{Raman spectra of silver nanoparticles}

Fig.6 shows SERS spectra of R6G molecules $\left(10^{-6} \mathrm{~mol} / \mathrm{L}\right)$ adsorbed on the same volume of silver nanoparticles for three heating time ( $t=8,15,20 \mathrm{~min})$. In Fig.6 the solid curve exhibits SERS spectrum of R6G molecules adsorbed without silver nanoparticles. We find that the Raman enhancement signals are very weak, and Raman peaks of R6G molecules nearly disappear. In order to demonstrate the SERS enhancement of silver nanoparticles, the silver nanoparticles were added. The other curves in Fig.6 show SERS spectrum of R6G molecules adsorbed with three kinds of silver nanoparticles. Fundamental Raman peaks of R6G molecules appear and exhibit the strongest SERS signal with adding silver nanoparticles at reaction time $15 \mathrm{~min}$. For R6G molecules, the characteristic stretching and bending vibrations has been
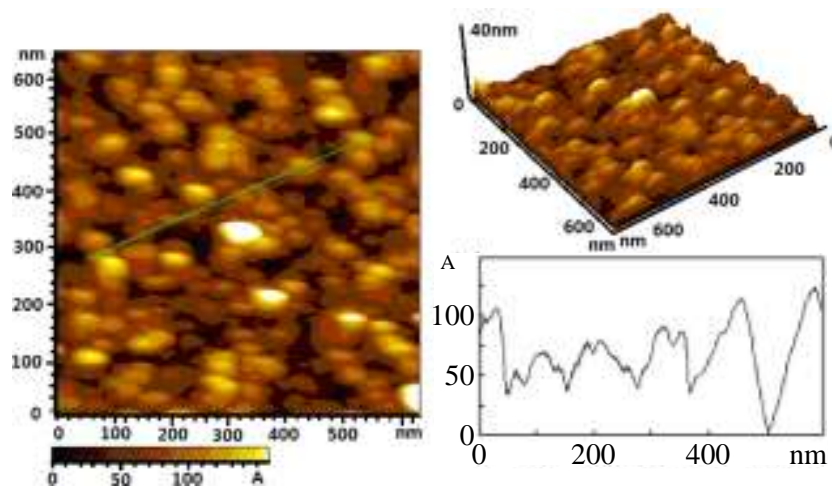

Fig.5 AFM images of silver nanoparticles prepared under microwave irradiation for $15 \mathrm{~min}$

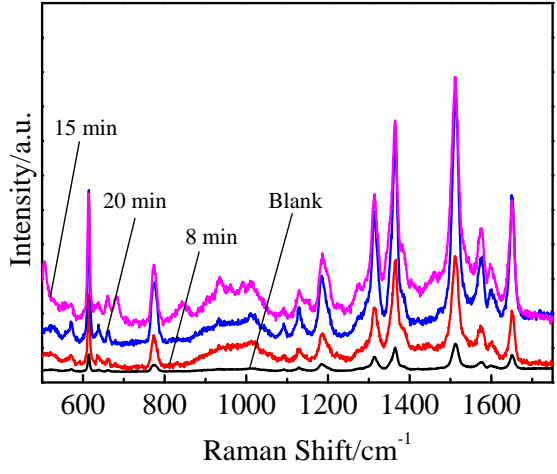

Fig.6 SERS spectra of R6G adsorbed on different silver nanoparticles prepared for different heating time

assigned as C-C-C ring in plane vibration $\left(618 \mathrm{~cm}^{-1}\right), \mathrm{C}-\mathrm{H}$ out of plane vibration $\left(773 \mathrm{~cm}^{-1}\right)$, aromatic stretch vibrations of the chromophore (1365 and $1650 \mathrm{~cm}^{-1}$ ) and C-O-C streth vibrations $\left(1512 \mathrm{~cm}^{-1}\right)^{[18,19]}$. Obviously the whole Raman intensity of R6G adding silver nanoparticles is stronger than that without silver nanoparticles. The relative Raman intensity peak at 618,1365 and $1512 \mathrm{~cm}^{-1}$ is higher than the others, which could be attributed to the silver nanoparticles. It could be also concluded that the SERS is enhanced by silver nanoparticles, and the one with reaction time $15 \mathrm{~min}$ is better than that with reaction time $8 \mathrm{~min}$ and $20 \mathrm{~min}$.

It is widely accepted that the SERS enhancement is the result of a combination of electromagnetic effect and chemical effect. The electromagnetic effect results from the enhancement of the local electromagnetic field due to surface plasmon resonance of nanoscale surface roughness features ${ }^{[20]}$. Compared with other two kinds of silver nanoparticles, a more uniform silver nanoparticles size could be desirable by the silver nanoparticles at reaction time $15 \mathrm{~min}$. This leads to the presence of more hot spots. Therefore, it results in large local electromagnetic field enhancements in these SERS hot areas. Of course, the chemical effect also plays a very important rule in improving the SERS enhancement ${ }^{[21]}$. The R6G molecule is coordinated to the silver surface by means of the nonbonding electrons of the nitrogen atom in ethylamino group. The fluorescence energy of R6G is transported from the molecules to the metal surface, which lead to the reduction of the fluorescence intensity and amplification of the Raman enhancement factors $^{[22]}$. Therefore, the enhanced Raman vibration spectra of R6G molecules adsorbed on silver nanoparticles are the surface interaction process. Therefore, silver nanoparticles with reacting for $15 \mathrm{~min}$ have uniform particle size and good dispersion (Fig.4), which makes it easier to combine with the lone pair electrons of the R6G molecule. The existence of surface residues after the synthesis might significantly interfere the surface interaction. This explanation is consistent with charge transfer theory widely accepted by many researchers ${ }^{[23]}$. However, detailed theory of 
the chemical mechanism is unclear by far. Hence, further experiments as well as theoretical studies should be made to obtain a comprehensive understanding of the chemical mechanism in the following work.

\section{Conclusions}

1) We adopted the microwave irradiation method to obtain silver nanoparticles rapidly.

2) With the increasing of the reaction time, the size of silver nanoparticles increases.

3) The intensity of surface enhancement Raman scattering (SERS) signals could be increased by silver nanoparticles.

4) The silver nanoparticles reacted for $15 \mathrm{~min}$ give the strongest SERS signal. The size and distribution of silver nanoparticles play a very important role in improving the SERS signal. This method may open up more effective and convenient ways to make high SERS-active substrate.

\section{References}

1 Fleischmann M, Hendra P J, McQuillan A J. Chemical Physics Letters[J], 1974, 26: 163

2 Zhou J, Xu S P, Xu W Q et al. Journal of Raman Spectroscopy [J], 2009, 40: 31

3 Xie Z G, Lu Y H, Wang P et al. Chinese Physics Letters[J], 2008, 25: 4473

4 Richard B J C, Wang J, Tantra R et al. Faraday Discussions[J], 2006, 132: 201

5 Anjali Pal, Tarasankar Pal. Journal of Raman Spectroscopy[J], 1999, 30: 199

6 Li X Y, Xia Y X, Zhan L et al. Chinese Physics Letters[J], 2008, 25: 2140
7 Zhang J T, Li X L, Sun X M et al. The Journal of Physical Chemistry $B[\mathrm{~J}], 2005$, 109: 12544

8 Lesuffleur A, Kumar L K S, Brolo A G et al. The Journal of Physical Chemistry C[J], 2007, 111: 2347

9 Addison C J, Brolo A G. Langmuir[J], 2006, 22: 8696

10 Zhang C L, Wang K J, Han D J et al. Spectrochimica Acta Part A: Molecular and Biomolecular Spectroscopy[J], 2014 122: 387

11 Qian X M, Nie S M. Chemical Society Reviews[J], 2008, 37: 912

12 Xue J Y, Liu X H, Wang X. Rare Metal Materials and Engineering[J], 2012, 41(9): 1672 (in Chinese)

13 Kneipp J, Kneipp H, Rice W L et al. Analytical Chemistry[J], 2005, 77: 2381

14 Navaladian S, Viswanathan B, Varadarajan T K et al. Nanotechnology[J], 2008, 19: 045603

15 Zhang Z X, Shao G X, Liu H R. Rare Metal Materials and Engineering $[\mathrm{J}], 2013,42(4): 809$ (in Chinese)

16 Mohamed M B, AbouZeid K M, Abdelsayed V et al. ACS Nano [J], 2010, 4: 2766

17 Abdelsayed V, Aljarash A, El-Shall M S et al. Chemistry of Materials[J], 2009, 21: 2825

18 Lee P C, Meisel D. The Journal of Physical Chemistry[J], 1982, 86: 3391

19 Majoube M, Henry M. Spectrochim Acta[J], 1991, 47: 14591

20 Moskovits M. The Journal of Physical Chemistry[J], 1978, 69: 1459

21 Ding S Y, Wu D Y, Yang Z L et al. Chemical Journal of Chinese Universities[J], 2008, 29: 2569

22 Tiwari V S, Oleg T, Darbha G K et al. Chemical Physics Letters [J], 2007, 446: 77

23 Quagliano L G. Journal of the American Chemical Society[J], 2004, 126: 7393

\title{
微波辅助法制备纳米银胶及其在拉曼表面增强方面的应用
}

\author{
刘发现 ${ }^{1,2}$, 刘 杰 $^{1}$, 曹雪玲 ${ }^{3}$
}

(1. 北京化工大学 碳纤维及功能高分子教育部重点实验室, 北京 100029)

(2. 中国石油吉林石化公司, 吉林 吉林 132002)

(3. 吉林化工学院, 吉林 吉林 132022)

摘 要: 采用一步微波辅助法制备了纳米银胶, 并利用紫外-可见吸收光谱, 透射电子显微镜（TEM）和原子力显微镜（AFM）等检测 手段对纳米银胶相应的性质和结构进行了表征。利用罗丹明 $6 \mathrm{G}$ (R6G) 为探针分子，考察了不同反应时间制备的纳米银胶对拉曼散射 (SERS) 的表面增强效果。结果表明, 纳米银胶可以提高拉曼散射的信号强度, 比较和分析了增强后的拉曼光谱。同时, 对纳米银胶 增强拉曼散射信号的机理进行了详细的研究。

关键词: 微波辅助法; 纳米银胶; 拉曼表面增强

作者简介: 刘发现, 男, 1981 年生, 博士, 北京化工大学碳纤维与复合材料研究所, 北京 100029, 电话: 010-64438724, E-mail: carbon-00@163.com 